\title{
Heat flow measurement in thin films by time resolved ellipsometry
}

\author{
H. El Rhaleb, J.P. Roger, J.L. Stéhlé* and A.C. Boccara \\ Laboratoire d'Optique Physique, UPR A0005 du CNRS, ESPCI, 10 rue Vauquelin, 75005 Paris, France \\ * SOPRA, 26 rue P. Joigneaux, 92270 Bois-Colombes, France
}

\begin{abstract}
Time resolved ellipsometry is applied to investigate heat diffusion processes occurring in polymer coated silicon under pulsed excitation. Experimental results, modelisation and analysis are presented.
\end{abstract}

\section{INTRODUCTION}

In a companion paper [1] we have demonstrated the ability of time resolved ellipsometry to monitor the surface temperature evolution of a bulk sample following the absorption of a light pulse. In the present study we show that a precise time resolved analysis of heat diffusion through a transparent layer can be achieved.

We have previously emphasised that stimulated ellipsometry can provide more pertinent parameters than photoreflectance. Indeed for micron thick films of low thermal conductivity such as polymer coatings deposited on silicon wafers, this method enables us to distinguish dynamic processes occurring in the substrate and in the film. On one hand, the refractive index variation of the substrate, on the other hand the heat diffusion through the film can be clearly separated by quantitative analysis of the stimulated ellipsometry data.

\section{RESULTS AND DISCUSSION}

As it is already mentioned in the other paper [1], this technique relies on the use of a short duration pulse (30ns of doubled YAG laser at $0.53 \mu \mathrm{m}$ ) to produce a heat source in a sample (spot diameter $\approx 1 \mathrm{~mm})$; the temperature field is then probed by another polarized light beam $(0.67 \mu \mathrm{m}$ diode laser, spot diameter $\approx 50 \mu \mathrm{m}$ ) which is reflected at the sample surface. The decay vs time of ellipsometric parameters variations $\delta \operatorname{tg} \Psi$ and $\delta \cos \Delta$ contains informations about thermophysical and optical properties of the samples.

The results we choose to report have been obtained for two PMMA (poly(methyl methacrylate)) coated silicon wafers. The samples have been first characterized (refractive indices and thicknesses) and proved to be homogeneous by spectroscopic ellipsometry measurements. The thicknesses of polymer layers are 0.122 and $2.2 \mu \mathrm{m}$.

At the excitation wavelength, the polymer films are transparent, so that the heat source is created at the interface with the substrate (light penetration depth for silicon is $\approx 0.8 \mu \mathrm{m}$ at $0.53 \mu \mathrm{m}$ ).

Figure 1 shows a typical set of data recorded in a stimulated ellipsometry experiment. Photoinduced signals for eight azimuths of the analyser has been measured as a function of time for the thick film and normalised to the DC signal values. The ellipsometric parameters changes are calculated from four of them. We clearly notice that time evolution is strongly dependent on the analyser azimuth revealing different types of induced effects and diffusion processes. Especially, the signals for the polarization directions 0,135 and $155^{\circ}$ show an immediate decay after the end of the excitation pulse, while for $45,65,90$ and $110^{\circ}$ directions the signals exhibit a nearly constant value up to $10 \mu \mathrm{s}$. Such 


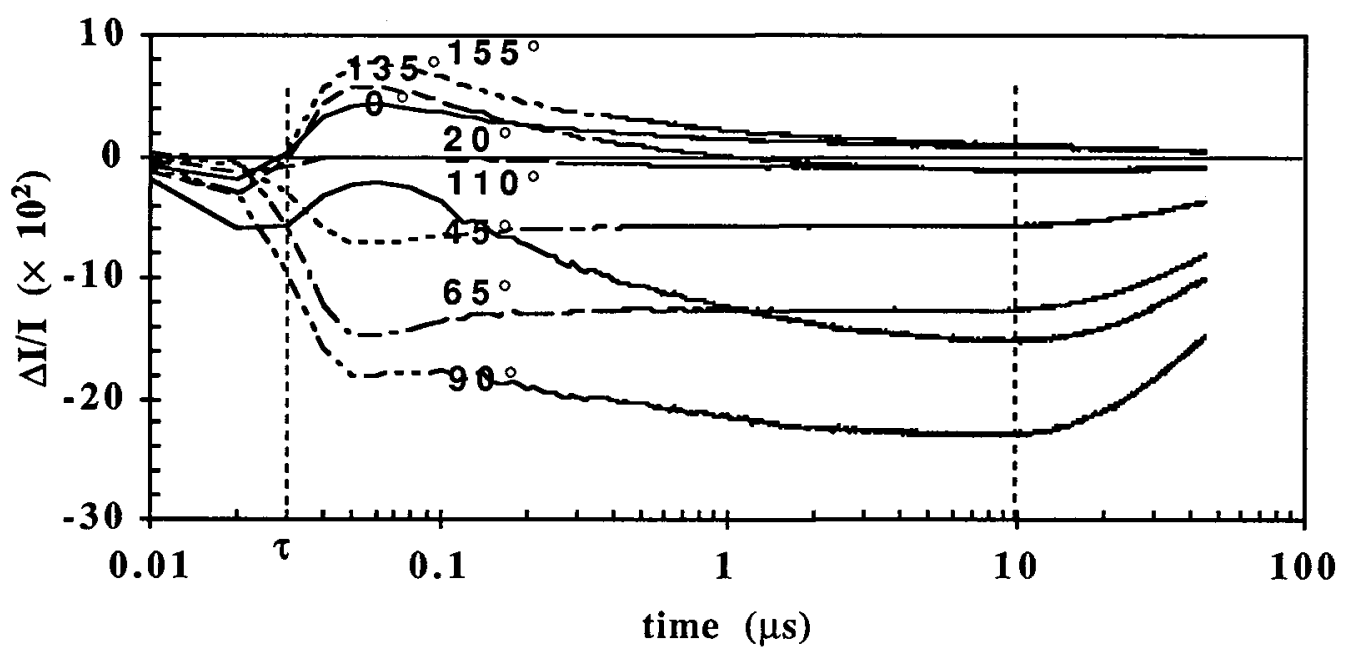

Fig. 1 - Relative photoinduced variation as a function time of the intensity reflected by the thick polymer film under pulsed excitation for eight azimuths of the analyser indicated on the curves. Azimuth of the polarizer : $45^{\circ}$, angle of incidence : $70^{\circ}$

results well illustrate the larger analytical ability of the stimulated ellipsometry method compared with the the one of the photoreflectance method.

The ellipsometric parameters variations for the two polymer coated wafers and a bare silicon wafer are reported in Fig. 2. The silicon wafer is covered by a $4 \mathrm{~nm}$ thick native oxide layer. Considering the high carrier injection $\left(\approx 10^{-21} \mathrm{~cm}^{-3}\right)$ in this experiment, the carrier recombination time is lower than $1 \mathrm{ps}$ and then the carrier effects on optical properties are only occurring during the light pulse. Later, the time response of the silicon sample (Fig. 2a) is governed by the one dimensional heat diffusion in the wafer (thermal diffusion length at $10 \mu \mathrm{s} \approx 60 \mu \mathrm{m}<$ spot size $\approx 1 \mathrm{~mm}$ ) as demonstrated by the $\mathrm{t}^{-1 / 2}$ behaviour of the ellipsometric parameters decay. For the probe beam $(0.67 \mu \mathrm{m})$, the optical penetration depth in silicon substrate $(\approx 3.7 \mu \mathrm{m})$ is smaller than the thermal diffusion length during the initial $50 \mu$ s $(\approx 4.3 \mu \mathrm{m})$. There'after, the temperature in the probed region of the substrate can be assumed to be homogeneous and to decrease like the interface temperature. The bulk sample data analysis described in the previous paper/1/, can be applied to the silicon sample result in order to lead to the surface temperature. For silicon at the probe wavelength, the real part $(n=3.8)$ of the refractive index is much larger than the imaginary part $(\mathrm{k}=0.014)$, so that the thermal effect is dominated by the real part variation $\left(\delta \mathrm{n} / \delta \mathrm{T} \approx 610^{-3}{ }^{\circ} \mathrm{C}^{-1}\right)$.

For the polymer coated wafers, the short time evolution of the ellipsometric parameters should be related to the heat diffusion through the polymer films. Indeed if the thermal diffusion time inside the film is longer than the time resolution of the detection $(10 \mathrm{~ns})$, then signals can reveal the heat diffusion processes. For the thinest film (Fig. 2b), a slope change occurs at about $0.1 \mu$ s corresponding to a thermal diffusion length of the order of the film thickness.

If we focus our attention on the time evolution of $\delta \operatorname{tg} \psi$ for the thickest film (Fig. 2c), we notice that $\delta \operatorname{tg} \psi$ is constant up to $\approx 10 \mu$ s corresponding to complete heat diffusion through the coating thickness. At late times the signal decay is due to diffusion into silicon substrate characterized by a $t^{-1 / 2}$ evolution. Indeed using one dimensional expression of the thermal diffusion length $\sqrt{4 \mathrm{D}_{\mathrm{f}} \mathrm{t}}$, and the literature value of the PMMA thermal diffusivity $\left(D_{\mathrm{f}} \approx 1.210^{-3} \mathrm{~cm}^{2} / \mathrm{s}\right)$, at $\mathrm{t}=10 \mu \mathrm{s}$ we get $2.2 \mu \mathrm{m}$ which is in good agreement with the value of the film thickness determined by spectroscopic ellipsometry.

The different temporal behaviours mentioned above in the measured signals (Fig. 1) can be also observed between the $\delta \operatorname{tg} \psi$, approximately constant, and $\delta \cos \Delta$ evolutions. The later exhibits a decay related to the temperature decrease of the surface substrate as shown in the following modelisation.

At times shorter than the thermal diffusion time through the layer, such as $\sqrt{4 D_{f} t}<$ thickness, the 

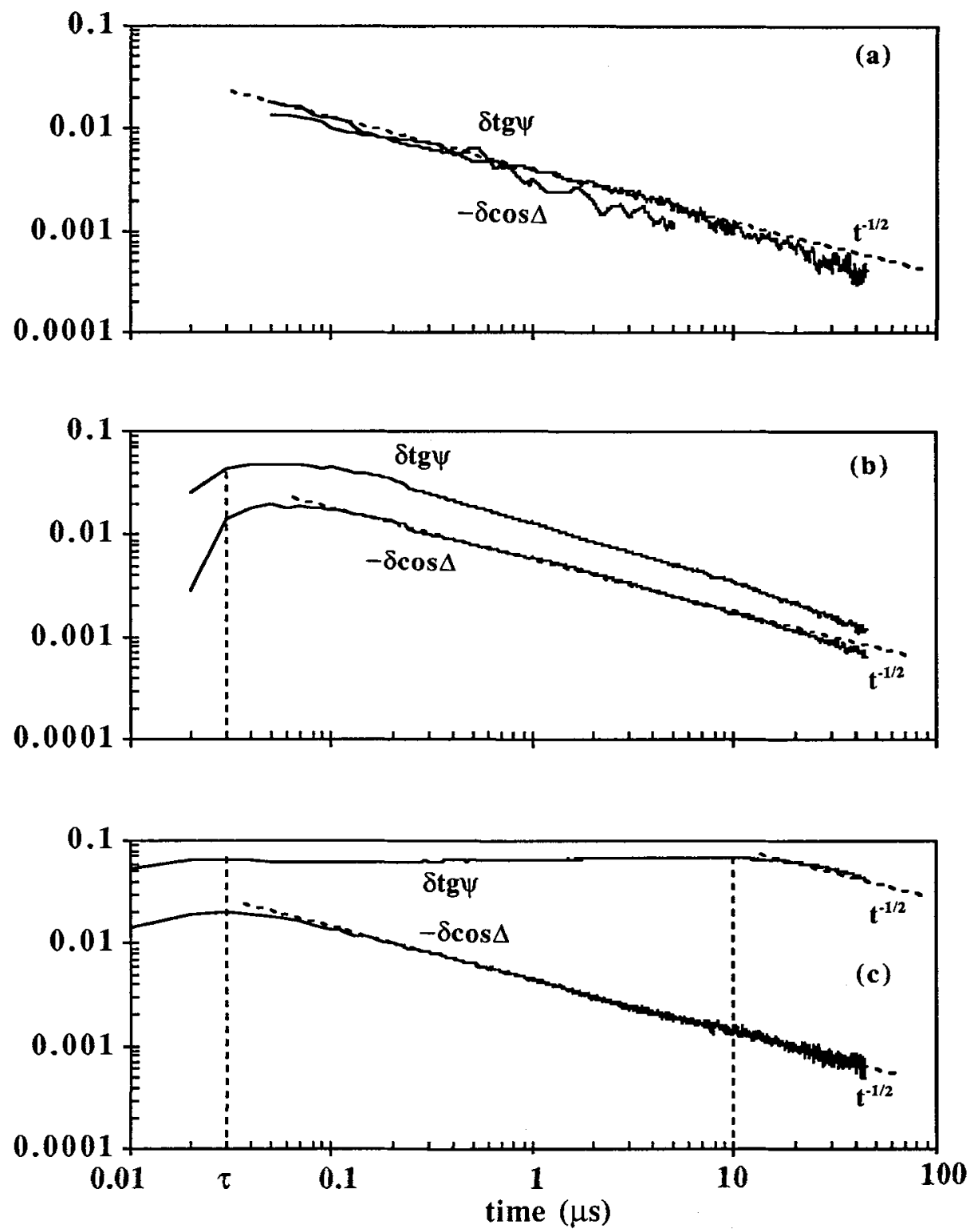

Fig. 2 - Time evolution of the ellipsometric parameters variations induced by a pulsed excitation $(0.53 \mu \mathrm{m})$ for a $0.67 \mu \mathrm{m}$ probe beam at the incidence angle of $70^{\circ}$ for three silicon wafers covered by: a) a $4 \mathrm{~nm}$ thick oxide layer, b) a $0.122 \mu \mathrm{m}$ thick PMMA film, c) a $2.2 \mu \mathrm{m}$ thick PMMA film.

temperature profile in the film is given within a good approximation by temperature solution of one dimensional heat diffusion equation in the case of a planar source at the interface $(x=0)$ of two semiinfinite media:

$$
\delta T(x, t)=\frac{Q}{\left(e_{f}+e_{s}\right) \sqrt{\pi t}} e^{\frac{-x^{2}}{4 D_{f} t}}
$$


where $Q$ is the deposited energy density, $e_{f}$ and $e_{s}$ are the thermal effusivities of the film and the substrate respectively.

Because a temperature change in polymer induces a refractive index change, the optical effect in the film is simply described by an index change profile proportional to the temperature one. This profile has been introduced in the calculation of the ellipsometric parameters as follows : the polymer layer is divided into ten sublayers and the refractive index value of each sublayer is obtained by averaging the index profile over the sublayer. Assuming an initial perturbation corresponding to an average value $\Delta \mathrm{n}=-510^{-3}$ in the first sublayer, we get the full curve presented in Fig. 3.

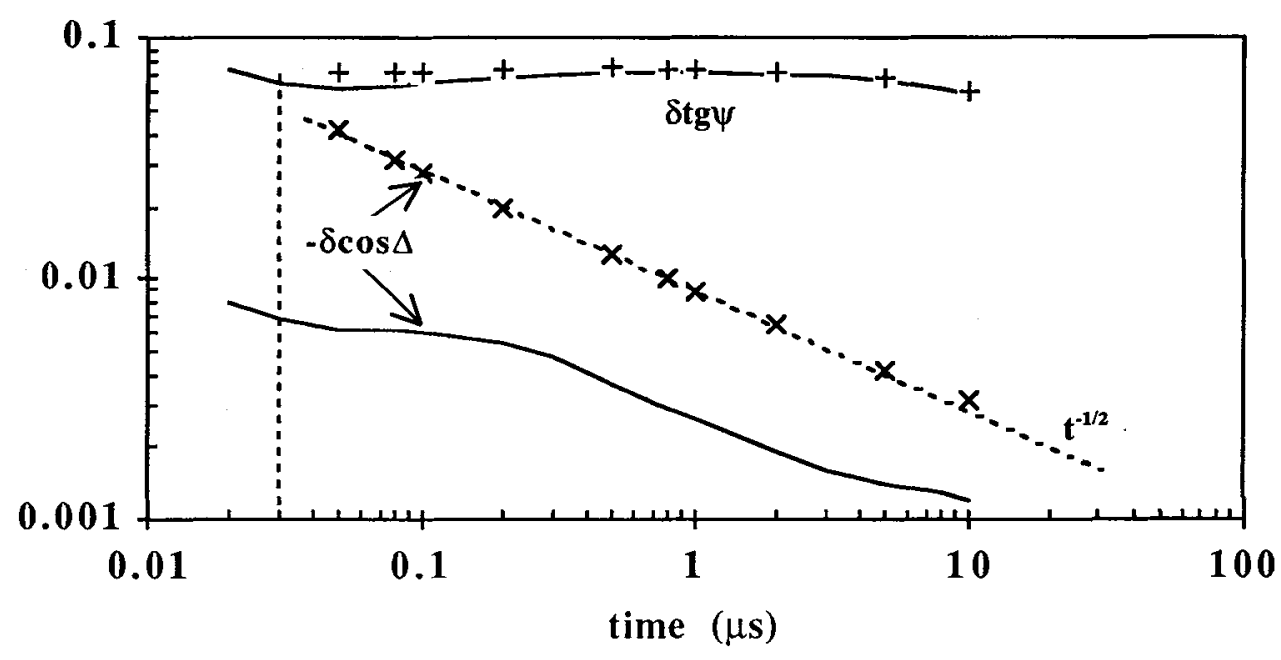

Fig. 3 - Theoretical calculation of the ellipsometric parameters variations vs. time for a $2.2 \mu \mathrm{m}$ thick polymer film on silicon:

full line: heat diffusion through the film corresponding to an initial index perturbation $\Delta \mathrm{n}=-510^{-3}$ is only considered.

$(+)$ the index variations of the silicon substrate are also taken into account.

The amplitude and evolution of $\delta \operatorname{tg} \psi$ are in quite good agreement with the experimental results but $\delta \cos \Delta$ values are one order of magnitude lower than the experimental ones. These calculated variations of $\cos \Delta$ are too weak to be considered as a significant effect. However, the induced optical effect in the substrate has to be taken into account. Therefore, the index variations deduced from the measurement performed on oxidized silicon wafer as mentioned above have been introduced in the theoretical model. The results are plotted in Fig. 3. The $\cos \Delta$ variations are mainly due to this effect and exhibit à $t^{-1 / 2}$ behaviour like in the experimental result, while the $\operatorname{tg} \Psi$ variations are unaffected by the substrate temperature changes. Finally, under the conditions of this particular experiment, $\delta \operatorname{tg} \Psi$ reveals the heat diffusion within the film, while $\delta \cos \Delta$ is related to the interface temperature

\section{CONCLUSION}

This experiment is the first demonstration of the ability of the photothermal ellipsometric techniques to follow the heat flux within a micronic film. We hope that such a method in which the optical field acts as a submicronic local probe in depth will stimulate further interesting studies

\section{REFERENCE}

[1] H. El Rhaleb and J.P. Roger, this conference 